\title{
Comparison of different methods for characterizing multi-walled carbon nanotubes
}

\author{
C. Gommes ${ }^{\mathrm{a}, *}$, S. Blacher ${ }^{\mathrm{a}}$, N. Dupont-Pavlovsky ${ }^{\mathrm{b}}$, C. Bossuot ${ }^{\mathrm{a}}$, M. Lamy $^{\mathrm{a}}$, A. Brasseur ${ }^{\mathrm{a}}$, \\ D. Marguillier ${ }^{\text {d }}$, A. Fonseca ${ }^{\text {c }}$, E. McRae $^{\text {b }}$, J. B.Nagy ${ }^{\text {c }}$, J.-P. Pirard ${ }^{\text {a }}$ \\ ${ }^{a}$ Laboratoire de Génie Chimique, Université de Liège, B6a, B-4000 Liège, Belgium \\ ${ }^{\mathrm{b}}$ Laboratoire de Chimie du Solide Minéral, UMR 7555, Université Henri Poincaré-Nancy 1, BP 239, 54506 Vandoeuvre-les-Nancy, France \\ ${ }^{\mathrm{c}}$ Laboratoire de Résonance Magnétique Nucléaire, FUNDP, 61 rue de Bruxelles, B-5000 Namur, Belgium \\ ${ }^{\mathrm{d}}$ Laboratoire de Chimie Inorganique Structurale, Université de Liège, B6a, B-4000 Liège, Belgium
}

Available online 5 June 2004

\begin{abstract}
Four complementary and independent methods are used to characterize nanotube samples: (i) TEM observation coupled with image analysis, (ii) nitrogen and krypton adsorption isotherm analysis, (iii) thermogravimetry and (iv) wide angle X-ray scattering. The methodology is discussed on the basis of two multi-walled carbon nanotube samples produced by the CCVD technique with very different reaction rates. It is shown that the total amount of deposited carbon is larger for the sample produced at the higher rate, that the fraction of nanotubes in the deposited carbon does not seem to be significantly different, but that the crystallinity of the nanotubes wall is larger for the sample produced at the lowest reaction rate.

(C) 2004 Elsevier B.V. All rights reserved.
\end{abstract}

Keywords: Carbon nanotubes; Image analysis; Gas adsorption; X-ray scattering; Thermogravimetry

\section{Introduction}

Carbon nanotubes were discovered as a byproduct of fullerene synthesis in 1991 by Ijima [1,2]. These novel materials consist in a variable number of graphene layers rolled coaxially into a cylinder of nanometric diameter.

Ideally a characterization method aims at determining both the amount of nanotubes present in a given sample and their size and physical characteristics. Nanotube-containing samples are often first investigated by means of electron microscopy which allows determining distribution of the nanotube diameter. Recently, our group has developed an image analysis method for such characterization [3]. However, the question always arises about the homogeneity of the samples and of the representativeness of the used images. If the heterogeneity of the sample is known to arise at a small scale, the characterization can be improved by considering a larger number of micrographs, but if the heterogeneity occurs over a larger scale, the sample characterization using

\footnotetext{
* Corresponding author. Tel.: +32 436629 51; fax: +32 43663545 .

E-mail address: cedric.gommes@ulg.ac.be (C. Gommes).
}

only microscopy is open to criticism. In this case, there is a need for macroscopic characterization tools.

In this paper, the following regularly used characterization tools are applied to characterize nanotube samples: (i) transmission electron microscopy (TEM), together with image analysis, (ii) nitrogen and krypton adsorption isotherm analysis, (iii) wide angle X-ray scattering and (iv) thermogravimetry. The results of each method are discussed and compared to each other in order to give a coherent picture of the sample.

The methodology is applied to two nanotube samples synthesized with very different reaction rates according to the catalytic chemical vapor deposition (CCVD) technique [4].

\section{Materials and methods}

\subsection{Synthesis of the samples}

The nanotube samples used were obtained according to the catalytic chemical vapor deposition (CCVD) technique [4]. A mass spectrometer positioned at the exit of the reactor 
Table 1

Synthesis conditions of samples S1 and S2

\begin{tabular}{lllcl}
\hline & $x_{0(-)}$ & $Q(1 \mathrm{STP} / \mathrm{min})$ & $r_{0}\left(\mathrm{~mol} \mathrm{C}_{2} \mathrm{H}_{4} / \mathrm{s}\right)$ & $m_{\text {carbon }}(\mathrm{g})$ \\
\hline S1 & 0.1 & 1 & $5.5 \times 10^{-5}$ & 0.73 \\
S2 & 0.3 & 3 & $27.4 \times 10^{-5}$ & 1.69
\end{tabular}

$x_{0}$ : molar fraction of ethylene in the feed gas, $Q$ : total feed gas flow, $r_{0}$ : initial reaction rate; $m_{\text {carbon }}$ : final amount of deposited carbon.

analyses the exhaust gas composition, which allows the real time monitoring of the reaction.

The synthesis is controlled by modifying the working temperature, the amount of catalyst, the total flow rate $Q$, and molar fraction of ethylene $x_{0}$, of the feed gas. Samples S1 and $\mathrm{S} 2$ were prepared by positioning $1 \mathrm{~g}$ of catalyst in the furnace at $700{ }^{\circ} \mathrm{C}$ with two different feed gas characteristics, as reported in Table 1 . After $20 \mathrm{~min}$ of reaction, the product is allowed to cool down under a helium atmosphere and is then taken out of the reactor.

Fig. 1 displays the amount of deposited carbon as a function of time during the synthesis of S1 and S2, as assessed from the hydrogen content of the exhaust gas. The production rate, corresponding to the slope of the curves, decreases in both cases during the reaction. After $20 \mathrm{~min}$ the reaction is almost finished. The initial reaction rate, $r_{0}$, is defined as the maximum value of the reaction rate. It must be noted that even if the reaction rate continuously decreases during the synthesis, a large fraction of the carbon is deposited at a rate close to $r_{0}$. The values reported in Table 1 show that $\mathrm{S} 1$ was produced at a rate approximately five times lower than S2. The reaction final extent, estimated as the total amount of deposited carbon is also much lower for S1 than for S2.

The samples were used as produced without any purification step. They are therefore expected to contain (i) nanotubes, (ii) a catalyst residue and (iii) possibly amorphous carbon.

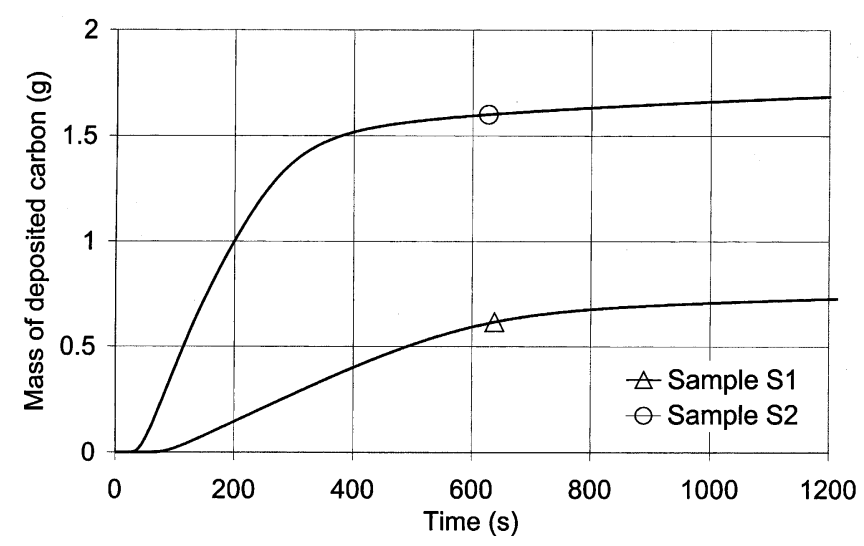

Fig. 1. Mass of deposited carbon as a function of time during synthesis of S1 and S2, as assessed from the mass spectrometer analysis of the exhaust gas.

\subsection{Characterization tools}

For the TEM observation, the samples were ultrasonically dispersed in ethanol and a drop of the solution was deposited on a copper microscopy grid. The micrographs were obtained on a Siemens Elmiskop 102 microscope at a magnification of 46000 . The micrographs were digitized on a $550 \times 750$ matrix with 256 gray levels and a resolution of $0.79 \mathrm{~nm} /$ pixel. 30 micrographs from each sample were analyzed.

$\mathrm{N}_{2}$ adsorption isotherms were determined at $77 \mathrm{~K}$ on a Carlo Erba Sorptomatic 1990 apparatus. The samples were outgassed for at least $24 \mathrm{~h}$ at room temperature at a pressure lower than $10^{-5}$ mbar. $\mathrm{Kr}$ adsorption isotherms were determined manually using a volumetric method as described elsewhere [5].

The thermogravimetric measurements were obtained on a SETARAM device which includes an arm microelectronic balance, an oven and a system enabling sample handling under vacuum or gas flow. The thermogravimetric experiments were performed using a linear heating rate of $2.5^{\circ} \mathrm{C} / \mathrm{min}$ under an air flow.

The wide angle X-ray scattering spectra were recorded on a Siemens D5000 powder diffractometer, using $\mathrm{Cu} \mathrm{K \alpha}$ radiation.

\section{Results}

\subsection{TEM and image analysis}

When the samples are observed at a low magnification (not shown) some dark zones are visible on the micrographs but it is not possible to ascertain if it is catalyst residue, amorphous carbon or mere entanglement of nanotubes. At higher magnifications nanotubes can be observed. Fig. 2 displays two typical micrographs obtained from samples S1 and S2. The outer radii of the nanotubes range in both samples from approximately 4 to $12 \mathrm{~nm}$.

Thirty such micrographs from each sample were analyzed using the image analysis methodology fully described elsewhere [3] and only briefly presented hereafter. This procedure allows determining the outer and inner radii, $R_{\mathrm{O}}$ and $R_{\mathrm{I}}$, of the nanotubes, as well as a linear electron absorption coefficient by the wall material $k$. The characterization of the nanotubes is based on their intensity profiles. Measurable sections of nanotubes appear as cylinders with a given gray level distribution, as seen from Fig. 3a. A local maximum intensity is seen along the tube's axis, which corresponds to its inner hollow cavity. Going away from the axis, the intensity decreases and reaches a minimum in the wall, and eventually increases again as one leaves the tube (Fig. 3b). On the same figure, the solid line represents a model intensity obtained by assuming that the tube is a perfect hollow cylinder whose wall absorbs the electrons of the incident beam according to Lambert's law, with coefficient $k$. By 

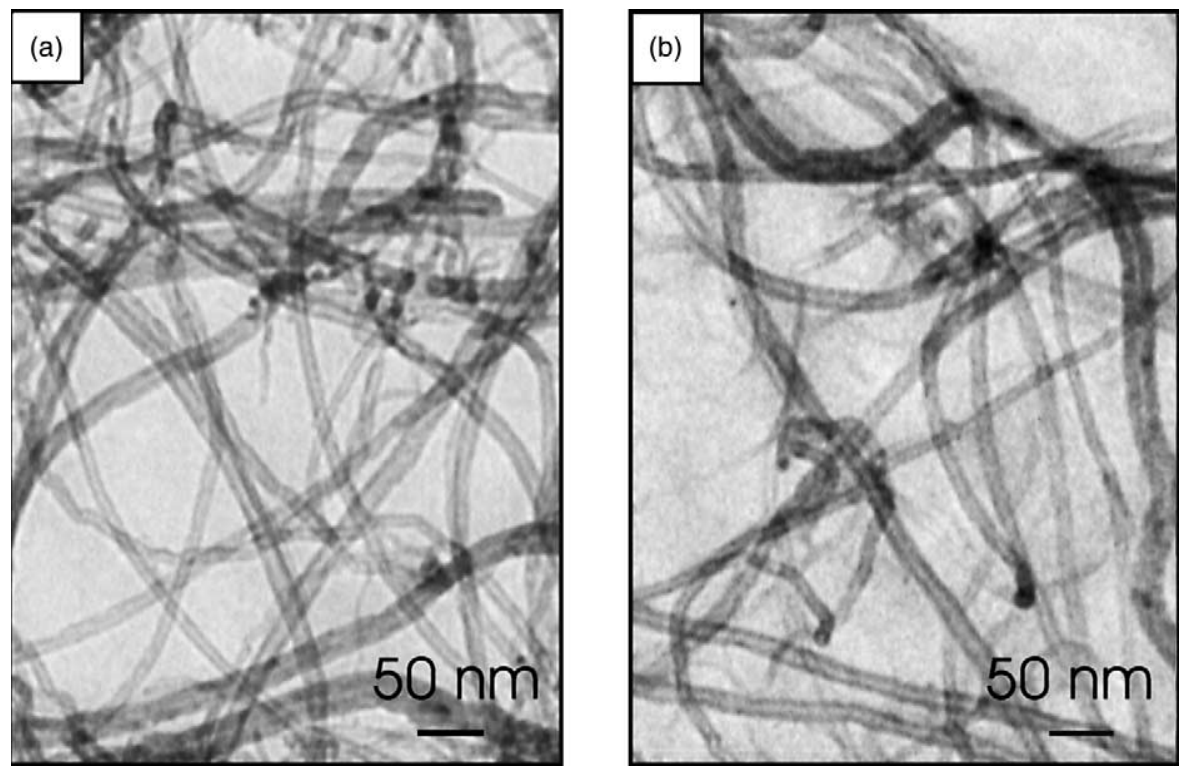

Fig. 2. Example of TEM micrographs of sample S1 (a) and S2 (b).

Table 2

Mean value and standard deviation of the nanotube characteristics estimated from image analysis

\begin{tabular}{lllll}
\hline & $R_{\mathrm{O}}(\mathrm{nm})$ & $R_{\mathrm{I}}(\mathrm{nm})$ & $T(\mathrm{~nm})$ & $k\left(1000 \mathrm{~nm}^{-1}\right)$ \\
\hline S1 & $7.2 \pm 2.3$ & $3.2 \pm 1.3$ & $4.0 \pm 1.8$ & $8.2 \pm 3.1$ \\
S2 & $7.3 \pm 2.5$ & $3.1 \pm 1.4$ & $4.2 \pm 2.0$ & $4.7 \pm 2.3$ \\
\hline
\end{tabular}

$R_{\mathrm{O}}$ : outer radius; $R_{\mathrm{I}}$ : inner radius, $T$ : wall thickness; $k$ : linear electron absorption coefficient.

fitting every determined intensity profile with this theoretical function, the statistical distributions of the outer and inner radii and of $k$ is obtained.

The estimated distributions of the characteristic parameters are represented in Fig. 4 and their mean values and standard deviations are reported in Table 2 . The inner and outer radii as well as the wall thickness of both samples are found to be very similar. However, S1 is characterized by a linear absorption coefficient which is twice as large as $\mathbf{S} 2$.

\subsection{Vapor adsorption isotherms}

\subsection{1. $\mathrm{N}_{2}$ adsorption}

Nitrogen adsorption isotherms of S1 and S2 are shown in Fig. 5a and $\mathrm{b}$. These do not correspond to any of the pure isotherms defined by the IUPAC classification [6]. Indeed, they have the following characteristics: (i) a narrow hysteresis loop is present for $P / P_{0}$ larger than 0.8 , (ii) in the mid pressure range, a slight convex tendency is visible on the isotherm of S2, as indicated by an arrow in the inset of Fig. 5b, which seems to be absent from sample S1, (iii) at very low pressures they resemble IUPAC type I isotherms.

In Fig. $5 \mathrm{c}$ and d, the adsorbed volume on both samples at a given pressure is plotted as a function of the statistical thickness $t$ of the $\mathrm{N}_{2}$ layer adsorbed at the same pressure on a reference non porous material. According to a procedure developed by Lecloux and Pirard [7], this reference is chosen on the basis of the interaction between the vapor and
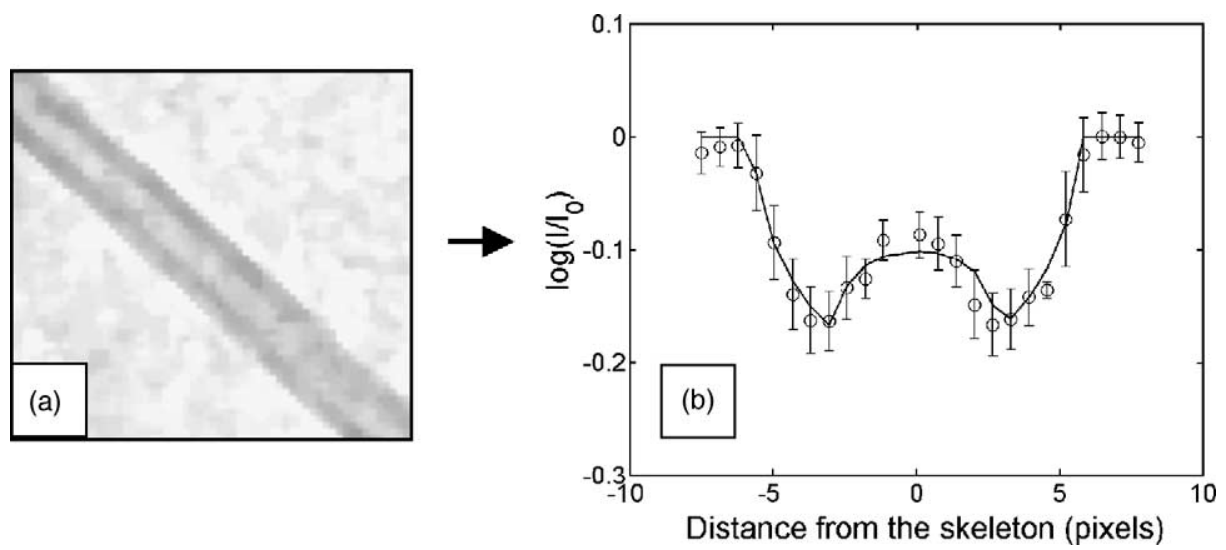

Fig. 3. Principle of nanotube characterization by the image analysis procedure: from a given nanotube (a), its intensity profile is determined as a function of the distance from its center (b). A least square fit allows $R_{\mathrm{O}}, R_{\mathrm{I}}$ and $k$ to be estimated for each selected tube in the images. 

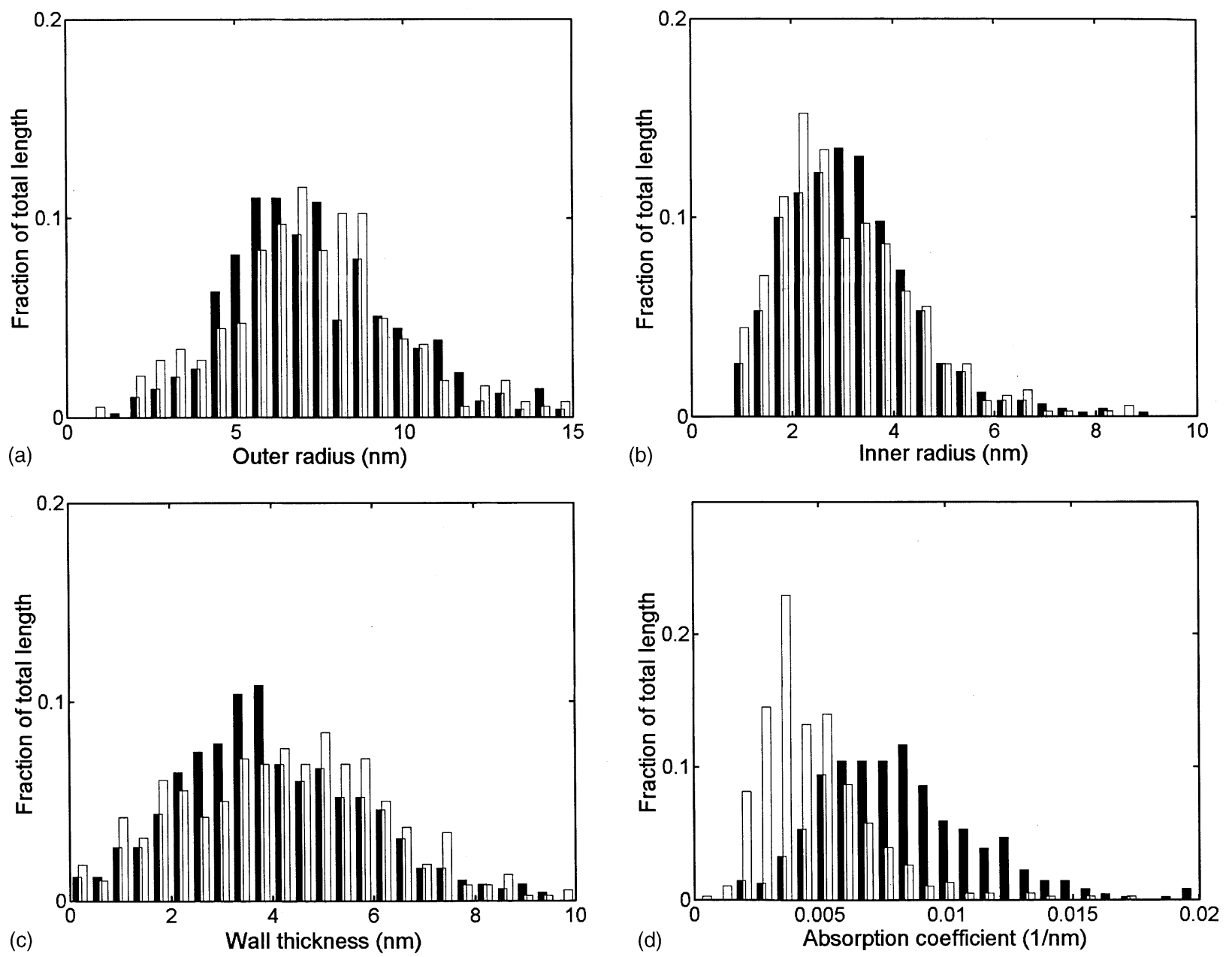

Fig. 4. Nanotube characteristic distributions estimated from image analysis, (a) outer radius, (b) inner radius, (c) wall thickness and (d) linear electron absorption coefficient, for samples S1 (black bars) and S2 (white bars).

the solid, as assessed by the value of the $C_{\mathrm{BET}}$ constant of the BET model. The $t$-plots corresponding to both samples exhibit a first linear trend whose slope $S_{t}$ is related to the samples specific surface area, followed by an upward deviation from linearity, which is much more visible for sample $\mathrm{S} 2$ than for $\mathrm{S} 1$, at a pressure corresponding to the hump in the isotherm of sample S2. At higher coverage, a second linear trend exists.

Table 3 reports the specific surfaces estimated from the BET model and the parameters of the linear parts of the comparison plots.

\subsection{2. $K r$ adsorption}

In the literature devoted to the comparative adsorption of different gases, the vapors are classified according to if they are $2 \mathrm{D}$-subcritical or 2D-supercritical at the considered temperature $[8,9]$. This refers to the possibility of 2-dimensional phase transitions in the adsorbed layers. No phase transition is observed for $\mathrm{N}_{2}$ at $77 \mathrm{~K}$, which is therefore referred to as supercritical. On the contrary, in the case of a 2D-subcritical vapors, such as $\mathrm{Kr}$ at $77 \mathrm{~K}$, a transition between a gas-like adsorbed phase and a liquid-like adsorbed phase occurs at a pressure independent of the surface coverage. This phenomenon occurs only on uniform and almost perfectly crystalline surfaces. This leads to a riser in the isotherms, which are classified as type VI according to IUPAC [6].

Fig. 6 shows the krypton adsorption isotherms determined on S1 and S2. On these isotherms, two steps are

Table 3

Nitrogen and krypton adsorption characterization of samples S1 and S2

\begin{tabular}{|c|c|c|c|c|}
\hline & \multicolumn{2}{|l|}{ S1 } & \multicolumn{2}{|l|}{ S2 } \\
\hline & $\mathrm{N}_{2}$ & $\mathrm{Kr}$ & $\mathrm{N}_{2}$ & $\mathrm{Kr}$ \\
\hline$S_{\mathrm{BET}}\left(\mathrm{m}^{2} \mathrm{~g}^{-1}\right)$ & 183 & 238 & 168 & 203 \\
\hline$S_{t}^{(1)}\left(\mathrm{m}^{2} \mathrm{~g}^{-1}\right)$ & 185 & $-^{\mathrm{a}}$ & 167 & $-^{\mathrm{a}}$ \\
\hline$S_{t}^{(2)}\left(\mathrm{m}^{2} \mathrm{~g}^{-1}\right)$ & 115 & $-^{\mathrm{a}}$ & 161 & $-^{\mathrm{a}}$ \\
\hline$V^{(2)}\left(\mathrm{cm}^{3} \mathrm{~g}^{-1}\right)$ & 56 & $-^{\mathrm{a}}$ & 40 & $-^{\mathrm{a}}$ \\
\hline$V_{\text {step }}\left(\mathrm{cm}^{3} \mathrm{~g}^{-1}\right)$ & $-^{\mathrm{a}}$ & 22 & $-{ }^{\mathrm{a}}$ & 31 \\
\hline$V_{\text {plateau }}\left(\mathrm{cm}^{3} \mathrm{~g}^{-1}\right)$ & $-^{\mathrm{a}}$ & 52 & $-^{\mathrm{a}}$ & 28 \\
\hline
\end{tabular}

$S_{\mathrm{BET}}$ : BET specific surface area, $S_{t}{ }^{(1)}$ : slope of the first linear trend in the $t$-plot, $S_{t}^{(2)}$ : slope of the second linear trend in the $t$-plot, $V^{(2)}$ : intercept of the second linear trend in the $t$-plot, $V_{\text {step }}$ : volume of the first adsorption step, $V_{\text {plateau }}$ : volume adsorbed in the plateau between the first two steps. ${ }^{\text {a }}$ Not measurable. 

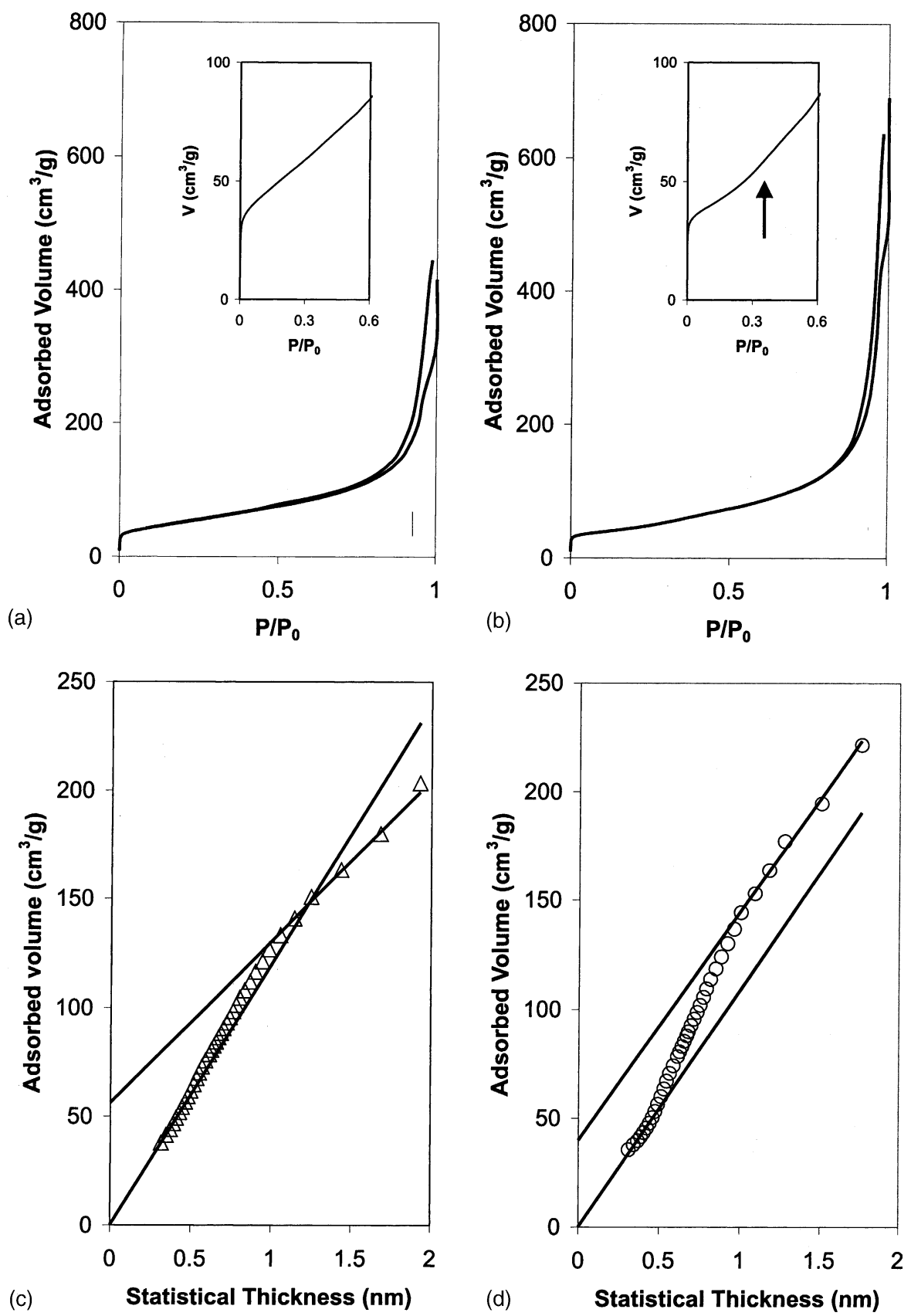

Fig. 5. Nitrogen adsorption isotherms of samples S1 (a) and S2 (b) and their corresponding comparison plots (c) and (d). The insets of figure (a) and (b) are a magnification of the low pressure part of the isotherms. The arrow indicates the Joyner-Emmet step of sample S2.

clearly visible: one at low relative pressures $\left(P / P_{0}=3 \times\right.$ $10^{-4}$ ), and the other near $P / P_{0}=0.5$. These steps were already reported in the literature on both multi-walled and single-walled nanotube samples $[5,10]$.

Table 3 summarizes the characteristics of the krypton adsorption isotherms. The BET specific surface area is obtained by taking $14.7 \AA^{2}$ as the molecular surface area of krypton. The amplitude of the first step, $V_{\text {step }}$, is determined as the amount adsorbed between the indicated arrows of Fig. $6 \mathrm{c}$ and d. The so-called plateau is defined as the range between the upper pressure limit of the first step and the lower pressure limit of the second step, indicated by an arrow in Fig. 6a and b.

\subsection{Thermogravimetry}

Fig. 7a shows the weight loss curves of samples $\mathrm{S} 1$ and S2 when they were submitted to a linear temperature change of $2.5^{\circ} \mathrm{C} / \mathrm{min}$ under an air flow. All the carbon of the samples burns below $550^{\circ} \mathrm{C}$, and the mass of the remaining ash at $700{ }^{\circ} \mathrm{C}$ is representative of the catalyst still present in the samples. The mass of the ash does not decrease 

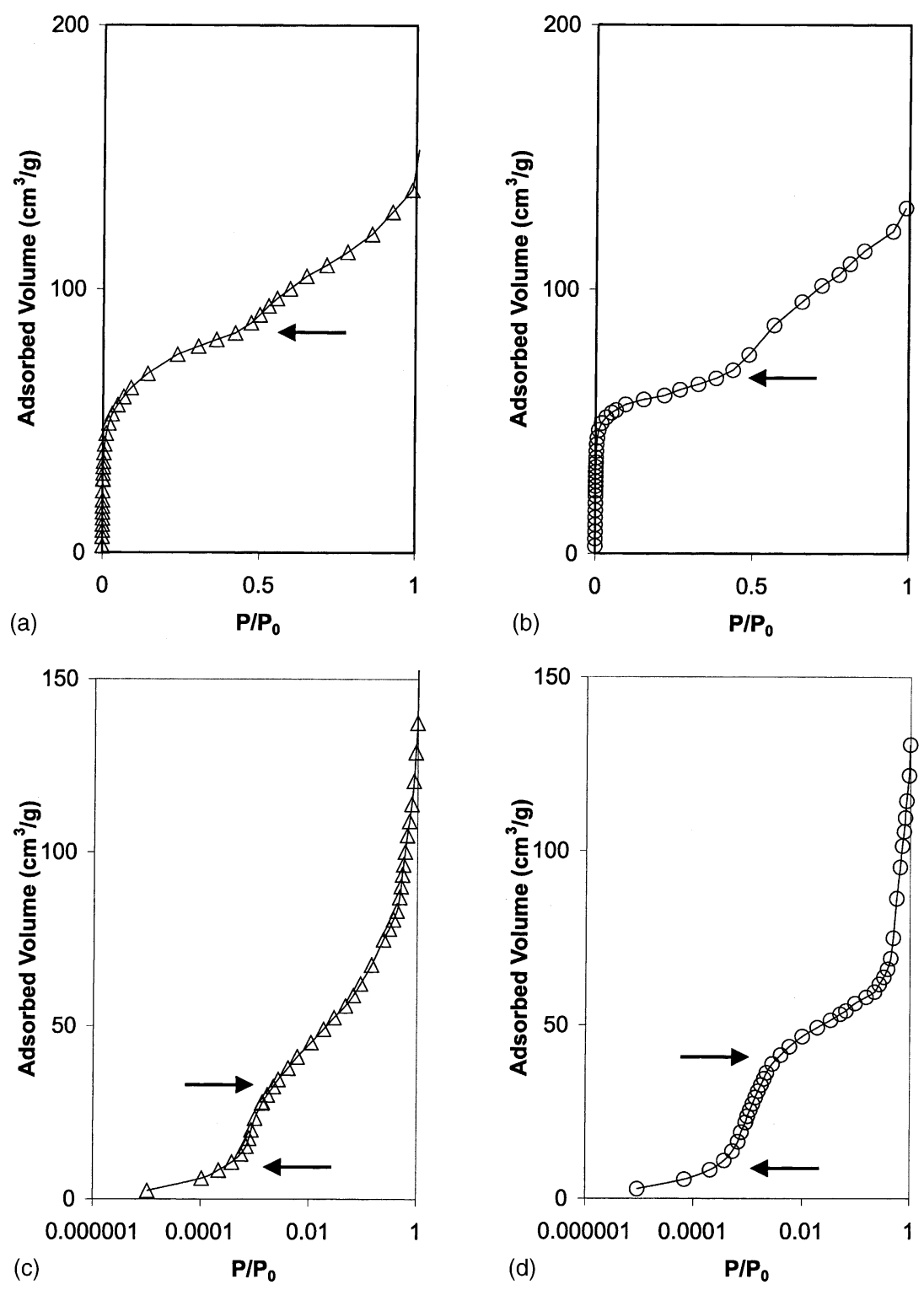

Fig. 6. Krypton adsorption isotherms of samples S1 (a and c) and S2 (b and d), subfigures c and d are a semi-logarithmic plot of a and b. The arrows in c and $\mathrm{d}$ indicate the lower and upper limits of the first condensation step and the arrow in a and $\mathrm{b}$ indicate the lower limit of the second condensation step.

anymore even when the temperature is further raised to $1000^{\circ} \mathrm{C}$.

A similar test performed on pure catalyst shows that it looses approximately $35 \%$ of its mass from 100 to $400{ }^{\circ} \mathrm{C}$. This loss is expected to be the weight of the hydration water.

By rescaling the sample masses to their burning content, an Arrhenius plot is drawn for both samples, which is shown

Table 4

Thermogravimetric characterization of samples S1 and S2

\begin{tabular}{llll}
\hline & Ash $(\%)$ & $E_{\mathrm{LT}}(\mathrm{kJ} / \mathrm{mole})$ & $E_{\mathrm{HT}}(\mathrm{kJ} / \mathrm{mole})$ \\
\hline S1 & 43 & 119 & 169 \\
S2 & 29 & 116 & 188 \\
\hline
\end{tabular}

Ash is the mass fraction of the sample that remains at $700^{\circ} \mathrm{C}, E_{\mathrm{LT}}$ : low temperature activation energy, $E_{\mathrm{HT}}$ : high temperature activation energy. in Fig. 7b. Two linear trends are visible, whose activation energies are reported in Table 4.

\subsection{Wide angle $X$-ray scattering}

Fig. 8a displays the X-ray diffraction spectra recorded from samples S1, S2 and from the catalyst. Characteristic

Table 5

X-ray diffraction characterization of samples S1, S2

\begin{tabular}{llllll}
\hline & $Q_{\mathrm{c}}\left(\AA^{-1}\right)$ & FWHM $\left(\AA^{-1}\right)$ & $d_{\mathrm{c}}(\AA)$ & $T(\AA)$ & $\Delta d_{\mathrm{c}}(\AA)$ \\
\hline S1 & 1.8258 & 0.2037 & 3.44 & 14.49 & 0.384 \\
$\mathrm{~S} 2$ & 1.8168 & 0.2536 & 3.46 & 11.63 & 0.484 \\
\hline
\end{tabular}

$Q_{\mathrm{c}}$ : position of the 002 peak, FWHM: full width at half maximum of the 002 peak, $d_{\mathrm{c}}$ : interlayer spacing, $T$ : thickness of the wall, $\Delta d_{\mathrm{c}}$ : FWHM of the interlayer spacings distribution. 

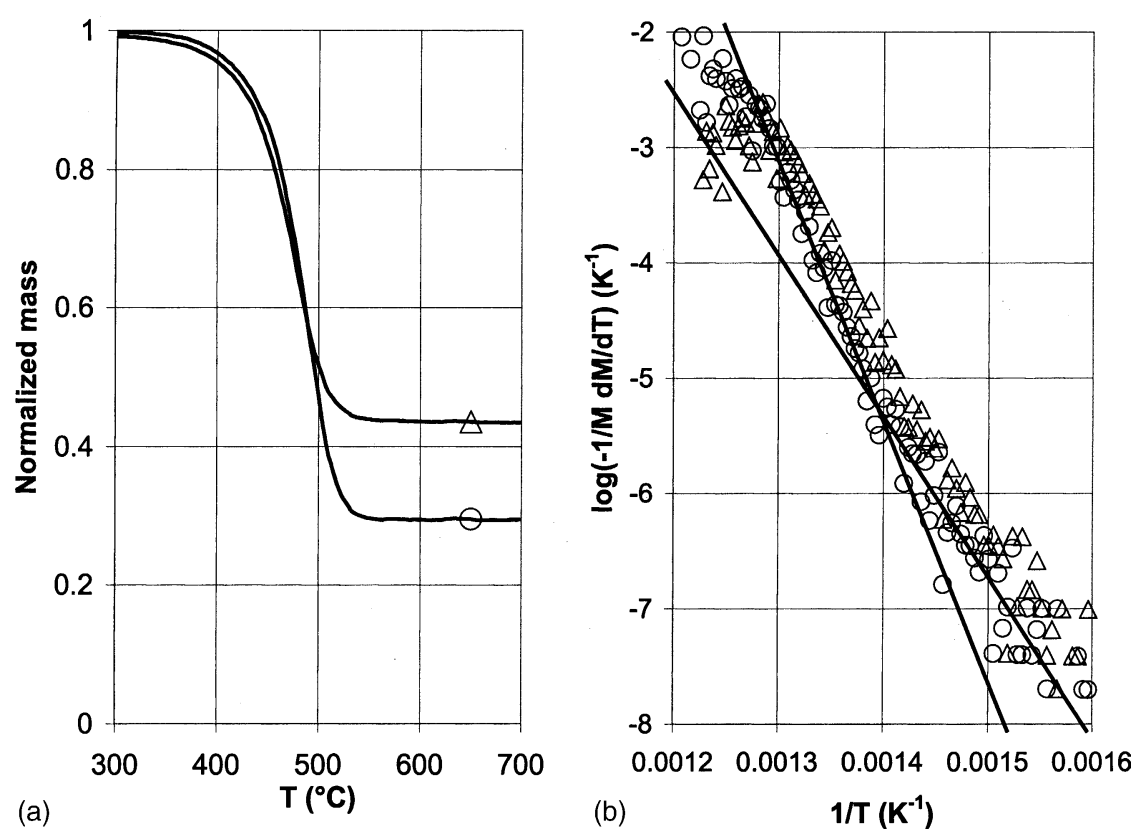

Fig. 7. (a) Weight loss curves of samples $S 1(\triangle)$ and $S 2(O)$, and (b) their corresponding Arrhenius plots showing two linear trends.

002 and 100 peaks of the graphite structure are visible [11]. These correspond respectively to the periodicity between the graphene layers and within the graphene layer. Other peaks are seen, which can be attributed to the catalyst.

The 100 peak is very sharply defined for S1 but barely visible in S2. Therefore, only the 002 peak is analyzed quantitatively in this paper. A Lorentzian curve is fitted to it by least squares (Fig. 8b), which allows its position and full width at half maximum (FWHM) to be determined precisely for both samples. The obtained values are reported in Table 5.

The position $Q_{\mathrm{c}}$ of the 002 peak allows the interlayer spacing to be determined. Its width can be interpreted in two different ways [11]: (i) the first possibility is the particle size broadening, which corresponds in the present case to the finite thickness of the nanotubes wall, (ii) the second is strain broadening, which results from a distribution of the interlayer spacings. In the case of size broadening, the FWHM is related to the thickness, $T$, of the wall by

$\mathrm{FWHM}=\frac{2 \sqrt{\pi \ln (2)}}{T}$

In the case of strain broadening, the corresponding relation is

$\mathrm{FWHM}=\frac{\pi l}{d_{\mathrm{c}}^{2}} \Delta d_{\mathrm{c}}$
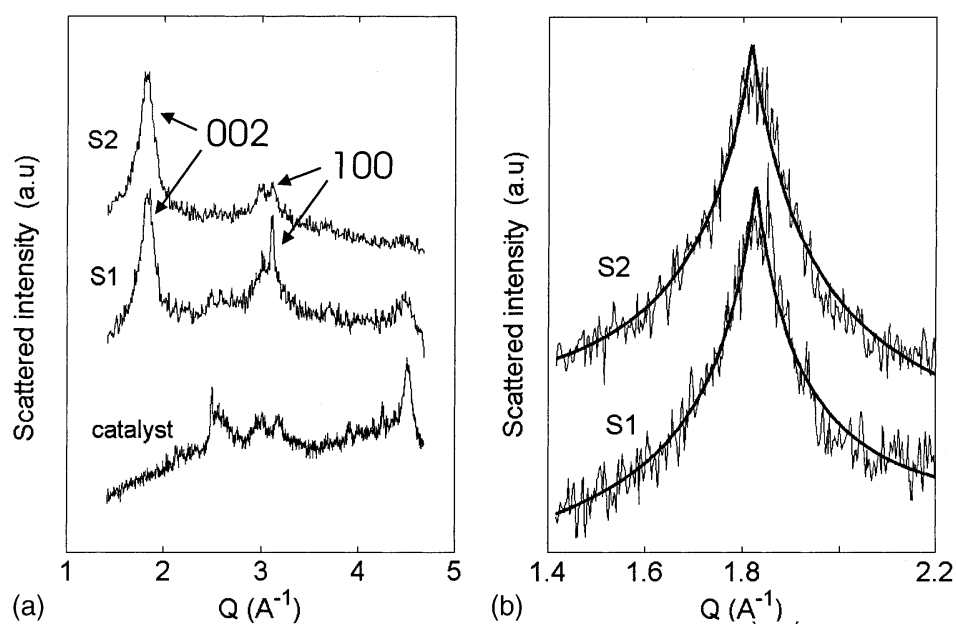

Fig. 8. Wide angle X-ray scattering spectra (a) of samples S1, S2 and of the catalyst. The 002 and 001 peaks typical of graphite-like structure are indicated by arrows. (b) Magnified view of the 002 peaks fitted by a Lorentzian model. 
where $l$ is the index of the reflection, and $\Delta d_{\mathrm{c}}$ is the FWHM of the $d_{\mathrm{c}}$ spacing distribution.

The quantities calculated from the spectra are given in Table 5. The choice between strain or size broadening will be discussed later.

\section{Discussion}

A complete characterization of a carbon nanotube samples implies determining (i) the mass fraction of the sample consisting of nanotubes, (ii) their geometric and physical characteristics. In this section, we discuss to what extent the characterization tools used allow these two goals to be achieved.

\subsection{Nanotube geometrical and physical characteristics}

Electron microscopy is definitely the most straightforward method for determining the size characteristics of the nanotubes. The image analysis procedure used makes such an analysis a less biased measurement than traditional eye made measurements. Using a Lambert law for the electron absorption by the wall material, a linear absorption coefficient is determined, which is not attainable from simple visual inspection of the micrographs. The most pronounced difference between $\mathrm{S} 1$ and $\mathrm{S} 2$ is in the value of $k$ which is two times larger for S1 than for S2. The numerical value of $k$ renders quantitative the observation that for a given wall thickness, one nanotube may appear more or less dark than another in the micrographs. The contrast in TEM images can be either diffusion contrast, in which a denser material appears darker, or diffraction contrast which makes the more ordered phases darker.

$\mathrm{X}$-ray scattering analysis confirms such a difference in the quality of the wall between the two samples. The average spacing between the graphene layers is found to be very close for both samples, but the FWHM of the 002 peaks are quite different. Since the nanotube wall thicknesses are found from TEM analysis to be similar for both samples, the differences in the FWHM of the 002 peak cannot be attributed to size broadening. Moreover, this interpretation would lead to an underestimation of the wall thickness as assessed by the values of $T$ reported in Tables 2 and 5. The differences in the FWHM must therefore result from strain broadening. The calculated value of $\Delta d_{\mathrm{c}}$ is found to be $25 \%$ larger for $\mathrm{S} 2$ than for $\mathrm{S} 1$, which means that the wall material in $\mathrm{S} 1$ is better crystallized. This interpretation is compatible with the values of $k$.

Thermogravimetric analysis could give information about the state of crystallinity of the wall material if the differences were sufficient for them to burn differently. For instance, Ketjen Black, a highly porous carbon black, burns with an activation energy close to $150 \mathrm{~kJ} / \mathrm{mol}$, while the activation energy for the burning of graphitic carbon is $206 \mathrm{~kJ} / \mathrm{mol}$ [12]. No such relationship was found between the burning characteristics of S1 and S2 and the aforementioned differences in the wall material. However, the difference between $E_{\mathrm{HT}}$ and $E_{\mathrm{LT}}$ is lower for $\mathrm{S} 1$ than for $\mathrm{S} 2$, which suggests that the burning material in $\mathrm{S} 1$ is more homogeneous.

As nanotubes have a well defined porosity, corresponding to their inner cavity, $\mathrm{N}_{2}$ adsorption would seem an appropriate tool for determining their inner diameters. However, the interpretation of these measurements is not straightforward. According to Inoue et al. [13] the interpretation of the comparison plot of S1 (Fig. 5c and Table 3) could be the following. The first linear trend corresponds to the surface coverage of both the inner and outer surface of the tubes and the second linear trend corresponds to the coverage of the outer surface. The same authors note however that Kelvin relation underestimates the actual size of the inner cavity of the nanotubes.

On the contrary, Yang et al. [14] showed that an acidic treatment, thought to remove the end tips of the nanotubes, enhances the low pressure part of the hysteresis at $P / P_{0}$ $=0.5-0.8$, which is therefore to be attributed to capillary condensation within the tubes.

The comparison plot of S2 (Fig. 5d) is quite different from $\mathrm{S} 1$. A much more pronounced upward deviation occurs and the specific surface is not significantly reduced afterwards. The deviation coincides with the hump visible on the isotherm itself, and indicated by an arrow in the inset of Fig. 5b. Since the hump in the $\mathrm{N}_{2}$ isotherm of $\mathrm{S} 2$ is not followed by any significant lowering in the available surface for further adsorption (Table 5), it can hardly be interpreted as the filling of some porosity. This result indicates that in the case of the considered samples, the nanotubes are closed, as already reported on other samples in the literature [14]. This is to be expected since the nanotubes were used as produced without any subsequent chemical treatment. As discussed in the following section, the adsorption isotherms on carbon nanotubes seem to give more information about their surface characteristics than about their porosity.

\subsection{Content of the samples}

\subsubsection{Carbon content}

If the carbonaceous species in the samples are supposed to be only nanotubes, with a negligible amount of amorphous carbon, estimating the amount of carbon in the samples is equivalent to estimating their purity. The two following methods can be used. (i) The first method is to divide the weighed mass of deposited carbon by the total mass of the sample containing both the "dry" catalyst and the carbon. When correction is taken for the $35 \%$ weight loss, resulting from the evaporation of hydration water in the reactor, the carbon content are found from the data of Table 1 to be $52 \%$ for $\mathrm{S} 1$ and $71 \%$ for $\mathrm{S} 2$. (ii) The second method is to use themogravimetry: from the weight of the ash (Table 4), the carbon content is found to be $57 \%$ for $\mathrm{S} 1$ and $71 \%$ for $\mathrm{S} 2$. 


\subsubsection{Nanotube content}

One key issue is to determine the amount of amorphous carbon in the samples. This type of carbon could be expected to burn differently than nanotubes. In this case thermogravimetric measurement could be useful. However, probably due to the presence of catalyst, the burning of the samples cannot be interpreted simply.

In order to evaluate the amount of nanotubes actually present in the samples another property typical of nanotubes has to exploited. For example, if some pore filling could be detected in the inner cavity of the tubes, this could serve as a measure of the nanotube content of the sample. However, as discussed previously, such a phenomenon cannot be ascertained with $\mathrm{N}_{2}$ adsorption.

Nevertheless a hump is visible in the $\mathrm{N}_{2}$ adsorption isotherms (Fig. 5b). Such a blunt step was already observed by Joyner and Emmet in the adsorption of $\mathrm{N}_{2}$ on Graphon [15]. It has since been observed by several authors for the adsorption of $\mathrm{N}_{2}$ on various well graphitized materials [16-18], and is usually referred to as the JE step. It is interpreted as the formation of the second nitrogen layer over a homogeneous surface. In that respect, it is interesting to compare the intercept of the second linear trend in Fig. 5d with the specific surfaces, estimated from the slopes of the linear parts. For nitrogen surface coverage, the adsorbed volume is related to covered surface by the following relation

$S_{\text {covered }}=4.352 V_{\text {adsorbed }}$

where $S_{\text {covered }}$ is expressed in $\mathrm{m}^{2} / \mathrm{g}$ and $V_{\text {adsorbed }}$ in standard $\mathrm{cm}^{3} / \mathrm{g}$. Using this relation, the amplitude of the JE step of S2 (estimated from the intercept of the second linear trend) is found to correspond to $174 \mathrm{~m}^{2} / \mathrm{g}$. This surface compares very well with the estimated specific surfaces (Table 3 ).

The shape of the $t$-plot of S1 (Fig. 5c) may result from the adsorption on the catalyst as well. Indeed, this sample contains a larger amount of catalyst residue than S2 and the JE step being not visible might be due to the fact that it is masked by the adsorption on this residue.

Concerning the $\mathrm{Kr}$ adsorption, in the ideal case of a perfectly uniform surface, the isotherm would consist of vertical steps separated by horizontal plateaus. We are not in this ideal case for the following two reasons. (i) Catalyst residue and amorphous carbon are present which produces a continuous adsorption. This is responsible for the high slope of the plateau. (ii) $\mathrm{Kr}$ condensation does not occur at once over the entire surface of the nanotubes, but occurs successively on several uniform patches with different characteristic energies [19]. The differences in the energies may result either from defects in the nanotubes wall or from the dispersion of their outer radii, i.e. from the curvature of the interfaces $[5,10]$.

Therefore, on the $\mathrm{Kr}$ adsorption isotherms of Fig. 6, only the surface of nanotubes contribute to the volume of the adsorption step, and mainly the catalyst and the amorphous carbon are responsible for the slope of the plateau. Strictly, a small amount of krypton adsorbs according to Henry's law on the nanotubes surface before the step. This corresponds to the formation of a $2 \mathrm{D}$ adsorbed gas. Nevertheless the density of this gaseous phase is much smaller than the 2D liquid phase which forms during the step. Consequently, the amplitude of the step is a good approximation of the volume adsorbed on the nanotube surface.

It is noteworthy (Table 4) that when the amplitude of the first step is normalized to the amounts of carbon known to be present in the samples, one finds $38.6 \mathrm{~cm}^{3} / \mathrm{g}_{\text {carbon }}$ for $\mathrm{S} 1$ and $43.7 \mathrm{~cm}^{3} / \mathrm{g}_{\text {carbon }}$ for $\mathrm{S} 2$. These volumes of adsorbed $\mathrm{Kr}$ are converted to surface by using the following relation

$S_{\text {covered }}=3.949 V_{\text {adsorbed }}$

where the surface covered by a single $\mathrm{Kr}$ molecule was estimated as $14.7 \AA^{2}, S_{\text {covered }}$ is expressed in $\mathrm{m}^{2} / \mathrm{g}$ and $V_{\text {adsorbed }}$ in standard $\mathrm{cm}^{3} / \mathrm{g}$. Using this formula, the specific surface of the patches on which $\mathrm{Kr}$ condensation occurs are found to be $152 \mathrm{~m}^{2} / \mathrm{g}_{\text {carbon }}$ for $\mathrm{S} 1$ and $172 \mathrm{~m}^{2} / \mathrm{g}_{\text {carbon }}$ for $\mathrm{S} 2$. From the TEM observation, the specific external surface area of the nanotube can be estimated as follows

$S=2 \frac{R_{\mathrm{O}}}{\rho\left(R_{\mathrm{O}}^{2}-R_{\mathrm{I}}^{2}\right)}$

where $\rho$ is the density of the wall material. Using the density of graphite, $\rho=2 \mathrm{~g} / \mathrm{cm}^{3}$, and the values reported in Table 2, this leads to the following two very close estimations of the external surface of the nanotubes: $173 \mathrm{~m}^{2} / \mathrm{g}_{\text {nanotube }}$ for $\mathrm{S} 1$ and $167 \mathrm{~m}^{2} / \mathrm{g}_{\text {nanotube }}$ for $\mathrm{S} 2$.

The comparison of the $\mathrm{Kr}$ adsorption and TEM analysis allows the following conclusions to be drawn: (i) since the normalized amplitudes of the condensation steps are very close for both samples $\left(152 \mathrm{~m}^{2} / \mathrm{g}_{\text {carbon }}\right.$ and $172 \mathrm{~m}^{2} / \mathrm{g}_{\text {carbon }}$ for S1 and S2 respectively), the amount of amorphous carbon in the samples is very similar. Indeed, should amorphous carbon be more present in a sample, this would not affect the absolute amplitude of the step, but would lower its amplitude normalized by the total amount of carbon (nanotubes and amorphous carbon). (ii) The surface corresponding to the krypton condensation step compares very well with the geometric external surface of the nanotubes. This confirms that the nanotubes are closed at both ends, as already noted when analyzing $\mathrm{N}_{2}$ adsorption. (iii) The previous two items suggest that the amount of amorphous carbon is very little in both samples. Indeed, by the nomalization mechanism discussed previously, the presence of amorphous carbon would lower the amplitude of the step below the geometric surface of the tubes. Furthermore, using the catalyst content determined from thermogravimetry (Table 4), one finds $121 \mathrm{~cm}^{3} / \mathrm{g}_{\text {catalyst }}$ for $\mathrm{S} 1$ and $96 \mathrm{~cm}^{3} / \mathrm{g}_{\text {catalyst }}$ for $\mathrm{S} 2$. Therefore, the amount of $\mathrm{Kr}$ adsorbed in the plateau between the steps are not very different for both samples, when normalized by the mass of catalyst. This confirms the absence of amorphous carbon in both samples since the amount of catalyst suffices to explain most of the adsorption in the plateaus. 
It must be noticed that the mere observation of the $\mathrm{Kr}$ isotherms of Fig. 6 could suggest either that the nanotube content of sample S2 is larger than S1, or that the surface of the nanotubes in S2 is more uniform than in S1. This last possible explanation is in contradiction with the conclusions of both X-ray and image analysis which prove that the nanotubes in sample $\mathrm{S} 1$ are better organized than in S2. On the other hand, the proper normalization of each part of the isotherms (condensation step and plateau) allows to conclude that the first assertion is correct and to further determine the composition of the impurities: mainly catalyst residue instead of amorphous carbon.

\section{Conclusion}

Typical multi-walled carbon nanotube samples produced by catalytic chemical vapor deposition usually consist in a blend of nanotubes, catalyst residue and amorphous carbon. When trying to characterize such samples, it is hard to assess the contribution of each of these components on the measured properties. This can only be achieved by performing independent characterization measurements and comparing their results. In this paper, TEM observation coupled with image analysis, $\mathrm{N}_{2}$ and $\mathrm{Kr}$ adsorption, thermogravimetry and $\mathrm{X}$-ray wide angle scattering were used to characterize two nanotube samples.

Image analysis and $\mathrm{X}$-ray diffraction allows geometrical and physical characteristics of the tubes to be determined. The electron absorption by the nanotube wall material, as assessed by image analysis, and X-ray diffraction lead to the same conclusion concerning the wall quality. Thermogravimetry measurement allows mainly the carbon content of the samples to be determined. The information given by both $\mathrm{N}_{2}$ and $\mathrm{Kr}$ adsorption measurements is related to the uniformity of the adsorbent surface. The amplitude of the $\mathrm{N}_{2}$ JE step can hardly be made quantitative, but in the case of $\mathrm{Kr}$, which is 2D-subcritical, the amplitude of the first condensation step can be measured and used to estimate the total external surface of the tubes. The so estimated surface compares well with the geometric surface estimated from TEM.

The proposed methodology was applied to two multi-walled carbon nanotube samples synthesized according to the
CCVD technique with very different carbon deposition rates. It has been shown that (i) the geometric characteristics of the nanotubes in both samples are similar (ii) the total amount of nanotubes produced is higher in the sample produced at the higher rate, (iii) the degree of crystallinity of the sample produced at the lowest rate is higher.

\section{Acknowledgements}

C. Gommes is grateful to the FNRS (National Fund for Scientific Research, Belgium) for a Ph.D. Research Fellow position. This work was supported by the Commission of the European Communities HPRN-CT-2000-00037 NANOCOMP contract, by the Communauté Française de Belgique 00/05-265 Action de Recherche Concertée, and by the Région Wallonne no. 0014622 grant.

\section{References}

[1] S. Ijima, Nature 354 (1991) 56.

[2] S. Ijima, Physica B 323 (2002) 1.

[3] C. Gommes, S. Blacher, K. Masenelli-Varlot, C. Bossuot, E. McRae, A. Fonseca, J. B.Nagy, J.-P. Pirard, Carbon 41 (2003) 2561.

[4] C. Journet, P. Bernier, Appl. Phys. A 67 (1998) 1.

[5] K. Masenelli-Varlot, E. McRae, N. Dupont-Pavlovsky, Appl. Surf. Sci. 196 (2002) 209.

[6] K. Kaneko, J. Membr. Sci. 96 (1994) 59.

[7] A. Lecloux, J.-P. Pirard, J. Colloid Interf. Sci. 70 (1979) 265.

[8] A. Thomy, X. Duval, J. Regnier, Surf. Sci. Rep. 1 (1981) 1.

[9] A. Thomy, X. Duval, Surf. Sci. 299 (1994) 415.

[10] M. Muris, N. Dufau, M. Bienfait, N. Dupont-Pavlovsky, Y. Grillet, J. Palmary, Langmuir 16 (2000) 7019.

[11] D. Reznik, C. Olk, D. Neumann, J. Copley, Phys. Rev. B 52 (1995) 116.

[12] R. Viswanath, P. Wilson, Eurasian ChemTech J. 3 (2001) 167.

[13] S. Inoue, N. Ichikuni, T. Suzuki, T. Uematsu, K. Kaneko, J. Phys. Chem. B 102 (1998) 4689.

[14] Q.-H. Yang, P.-X. Hou, S. Bai, M.-Z. Wang, H. -M Cheng, Chem. Phys. Lett. 345 (2001) 18.

[15] L. Joyner, P. Emmet, J. Am. Chem. Soc. 70 (1948) 2353.

[16] C. Pierce, B. Ewing, J. Phys. Chem. 68 (1964) 2562.

[17] P. Carrot, A. Roberts, K. Sing, Carbon 25 (1987) 59.

[18] K. Miura, H. Yanazawa, Carbon 41 (2003) 151.

[19] W. Rudzinski, D. Everett, Adsorption of Gases on Heterogeneous Surfaces, Academic Press, San Diego, 1992 (Chapter 8). 